\title{
Kaposi Sarcoma in HIV Infected Patients
}

\author{
Jipa Raluca, Săndulescu Oana, Manea Eliza, Benea Șerban, Benea Otilia, Hristea Adriana* \\ National Institute of Infectious Diseases "Prof. Dr. Matei Balș", Bucharest, Romania; "Carol Davila" University of Medicine and Pharmacy, Bucharest, Romania
}

Objective: The aim of the study was to describe clinical and laboratory characteristics in HIV-infected patients with Kaposi sarcoma (KS). Methods: We retrospectively studied data on HIV-infected patients hospitalized in one tertiary care hospital in Bucharest, Romania, in whom Kaposi Sarcoma was diagnosed, between January 2008 and November 2013. Results: We identified 27 HIV-infected patients diagnosed with KS within 6 years. They had a median age of 42 years old and a median CD4 cell count of 101 cells per $\mathrm{mm}^{3}$ at the time of KS diagnosis. All patients received antiretroviral therapy (ART), with 18 patients (66\%) already on ART at the time of KS diagnosis. Most patients (59\%) were classified as ACTG poor-risk and 56\% as Mitsuyasu stage I. The overall prognosis was poor, with 41\% mortality, in a median time span of 6 months, significantly correlated with gastrointestinal involvement ( $p=0.019)$, poor-risk KS in ACTG classification $(p<0.001)$ and stage IV Mitsuyasu ( $p=0.006)$. Conclusion: KS remains an important cause of morbidity and mortality in patients with HIV infection, especially in late presenters.

Keywords: Kaposi disease, HIV, late presenters

Received: 19 February 2016 / Accepted: 29 March 2016

\section{Introduction}

Kaposi sarcoma (KS) is a vascular tumor etiologically associated with human herpes virus 8 (HHV-8) [1]. KS was one of the first recognized Human Immunodeficiency Virus (HIV)-related illnesses in the early 1980s and is the second most frequent tumor associated with Acquired Immunodeficiency Syndrome (AIDS) [2]. KS's clinical course can range from minimal mucocutaneous lesions with or without associated extremity lymphedema to a rapidly progressing disease with frequent extracutaneous involvement of the mucous membranes, gastrointestinal tract and respiratory tract [3] that can lead to significant morbidity and mortality. Since the introduction of highly active antiretroviral therapy (HAART) the incidence of KS decreased dramatically, from $14.3 \%$ during 1980 1989 to $1.8 \%$ during 1996 - 2006 [4]. According to the European Center for Diseases Control (ECDC), between 2008-2013, 2132 cases of KS were reported among HIVinfected patients [5].

The aim of our study was to describe clinical and laboratory characteristics and to assess predictors for death in KS HIV-infected patients.

\section{Methods}

We performed a retrospective, observational study of HIVinfected patients hospitalized in one tertiary care hospital in Bucharest, Romania, in whom KS was diagnosed, between January 2008 and November 2013. The diagnosis of KS was based on physical examination and skin biopsy. To determine visceral involvement we used an individualized approach consisting of upper endoscopy, bronchoscopy and/or computed tomography.

* Correspondence to: Adriana Hristea
KS was staged according to the AIDS Clinical Trials Group (ACTG) [6] and the Mitsuyasu classification system [7]. According to the ACTG, KS was divided into goodrisk (noted by subscript 1 ) or poor-risk (noted by subscript 0 ) depending on the extent of the tumor (T0- KS confined to skin and/or lymph nodes and/or minimal oral disease; $\mathrm{T} 1$ - widespread lesions of KS) the status of the immune system (I0 - CD 4 cell count $\geq 200$ cells $/ \mathrm{mm}^{3}$; I1 - CD4 cell count $<200$ cells $/ \mathrm{mm}^{3}$ ) and the extent of involvement within the body or systemic illness (S0/S1).

In accordance to Mitsuyasu staging we stratified patients into: stage I-localized nodular KS, with $>15$ cutaneous lesions or involvement restricted to one bilateral anatomic site and few, if any, gut nodules; stage II-includes both exophytic destructive lesions and locally infiltrative cutaneous lesions as locally aggressive KS; stage III-generalized lymphadenopathic KS with widespread lymph node involvement, with or without skin lesions, but with no visceral involvement; stage IV-disseminated visceral $\mathrm{KS}$, usually progressing from stage II or stage III, with involvement of multiple visceral organs, and further into two subtypes: type $\mathrm{A}$ indicated the absence and type $\mathrm{B}$ the presence of "B" constitutional symptoms or opportunistic infections.

We used for prognostic a score proposed by Stebbing [8] in 2006: $\mathrm{KS}$ as the AIDS-defining illness (-3 points) and increasing CD 4 count $(-1$ point for every complete 100 cells $/ \mathrm{mm}^{3}$ ) improved diagnosis, age of 50 year or older ( 2 points) and having another AIDS-defining illness at the same time (3 points) were associated with poor prognosis.

The whole group was followed-up for two years. Patients lost to follow-up were not included in the survival analysis.

Statistical analysis was performed with SPSS v. 19.0 (Statistical Package for the Social Sciences Inc, IBM Corp, Armonk, NY, USA). The Mann-Whitney $U$-test was used to analyse the differences between groups for continuous 
variables. For categorical variables, the $\chi^{2}$ test or Fisher's exact test were used to test for differences between groups. The level of statistical significance was set at $\alpha=0.05$. Survival distribution was estimated using the Kaplan-Meier method.

\section{Results}

We identified 27 HIV-infected patients diagnosed with KS between January 2008 and November 2013. There was a male predominance in our study group, with an overall male to female sex ratio of 2:1. At the time of KS diagnosis the median CD4 count was 101 cells $/ \mathrm{mm}^{3}$ (IQR 41-270) and the HIV viral load was 151,704 copies/mL (IQR 92,317-398,500). Patient's characteristics are shown in table I.

HIV infection was diagnosed before KS in 19 patients (70\%), with a median time between HIV diagnosis and KS diagnosis of 19 months (IQR 0-58). In the remaining eight patients (30\%) KS was the first AIDS-defining illness.

Ten patients who were on ART, with a median duration of time between ART and KS diagnosis of 32 months had virological failure, due to non-adherence. The median CD4 count decreased from 200 cells $/ \mathrm{mm}^{3}$ at the time of HIV diagnosis to 52 cells $/ \mathrm{mm}^{3}$ at the time of KS diagnosis in this group. In the remaining nine patients who were on ART, the median CD4 count increased from a median of $200 \mathrm{cells} / \mathrm{mm}^{3}$ at the time of HIV diagnosis to 330 cells $/ \mathrm{mm}^{3}$ at the time of KS diagnosis, with a median duration of treatment of nine months. Eight out of nine (8/9) patients who were adherent to treatment and were on regimens with nucleoside reverse-transcriptase inhibitors (NRTIs) and protease inhibitors (PIs). Patients in whom $\mathrm{KS}$ was the first AIDS-defining illness had a median CD4 count of 62 cells $/ \mathrm{mm}^{3}$.

Regarding KS staging, $41 \%$ had T0 disease, $33 \%$ had I0 disease and 15\% had S0 disesease. Twenty-one patients (78\%) were in Mitsuyasu stage I and II.

Eleven patients $(41 \%)$ patients received topical steroid therapy, with a median duration of three months. Six (22\%) patients received specific treatment for KS: three local radiotherapy and three systemic therapy (two with interferon and one with liposomal doxorubicin).

The overall mortality was $41 \%(11 / 27)$, with a median duration between KS diagnosis and death of 6 months (IQR 2-15). Among patients who received specific treatment for KS, one (17\%) patient died, 15 months after the diagnosis of KS. The Kaplan-Meier estimates of the probability of survival were $77 \%(95 \%$ CI 57-90) at 6 months, 69\% (95\% CI 48-84) at 12 months and 64\% (95\% CI 44-81) at 24 months. Figure 1 shows the overall cumulative probability of survival of HIV infected patients with KS.

Gastrointestinal involvement ( $\mathrm{p}=0.019), \mathrm{CD} 4$ count $<200$ cells per $\mathrm{mm}^{3}(\mathrm{p}=0.042)$, poor-risk KS in ACTG classification $(\mathrm{p}<0.001)$ and stage IV Mitsuyasu $(\mathrm{p}=0.006)$ have been shown in univariate analysis to be associated with mortality (table II).

Table I. Patient's characteristics, clinical manifestations and staging

\begin{tabular}{lclll}
\hline Patient characteristics & N (\%) & Clinical manifestations & N (\%) & Staging \\
\hline Cutaneous KS localization & & Concomitant opportunistic infections & ACTG staging \\
Thorax & $2(7)$ & CMV retinitis & $4(15)$ & Good-risk KS \\
Facial & $2(7)$ & Oropharyngeal candidiasis & $7(26)$ & Poor-risk KS \\
Lower limbs & $16(59)$ & Pulmonary tuberculosis & $7(26)$ & Mitsuyasu classification system \\
Disseminated & $7(26)$ & Pneumocystosis & $4(15)$ & Stage I \\
Oral mucosal lesions & $10(37)$ & Cerebral toxoplasmosis & Stage II \\
Gastrointestinal involvement & $4(15)$ & Other malignancies & $1(4)$ & Stage III \\
Pulmonary involvement & $3(11)$ & Cerebral lymphoma & Stage IV \\
Male & $18(67)$ & Gastric lymphoma & $1(4)$ & Subtype A \\
Age(years) (median, IQR) & $42(34-52)$ & Pancreatic carcinoma & $1(4)$ & Subtype B
\end{tabular}

ACTG = AIDS Clinical Trials Group; CMV = cytomegalovirus; KS = Kaposi sarcoma

Table II. Clinical and laboratory characteristics associated with death

\begin{tabular}{|c|c|c|c|}
\hline & $\begin{array}{l}\text { Favorable outcome } \\
\qquad \mathrm{N}=16\end{array}$ & $\begin{array}{l}\text { Mortality related and unrelated to KS } \\
\qquad N=11\end{array}$ & p value \\
\hline Male, N (\%) & $8(50)$ & $10(91)$ & 0.042 \\
\hline Age (years) (median, IQR) & $43(35-52)$ & $37(21-61)$ & 0.604 \\
\hline KS-AIDS defining illness, $N(\%)$ & $4(25)$ & $4(36)$ & 0.675 \\
\hline Disseminated cutaneous lesions, N (\%) & $4(25)$ & $4(36)$ & 0.675 \\
\hline T1 stage, $\mathrm{N}(\%)$ & $9(56)$ & $7(64)$ & 1.000 \\
\hline S1 stage, N (\%) & $12(75)$ & $11(100)$ & 0.123 \\
\hline Gl involvement, N (\%) & $0(0)$ & $4(36)$ & 0.019 \\
\hline Pulmonary involvement, N(\%) & $0(0)$ & $3(27)$ & 0.056 \\
\hline Concomitant opportunistic infections, N (\%) & $6(38)$ & $7(64)$ & 0.103 \\
\hline ACTG poor-risk KS, N (\%) & $5(31)$ & $11(100 \%)$ & $<0.001$ \\
\hline Stage IV Mitsuyasu, N (\%) & $0(0)$ & $5(45)$ & 0.006 \\
\hline On ART, N (\%) & $16(100)$ & $8(73)$ & 0.056 \\
\hline
\end{tabular}

ACTG = AIDS Clinical Trials Group; ART = antiretroviral therapy; GI = gastrointestinal; KS = Kaposi sarcoma 


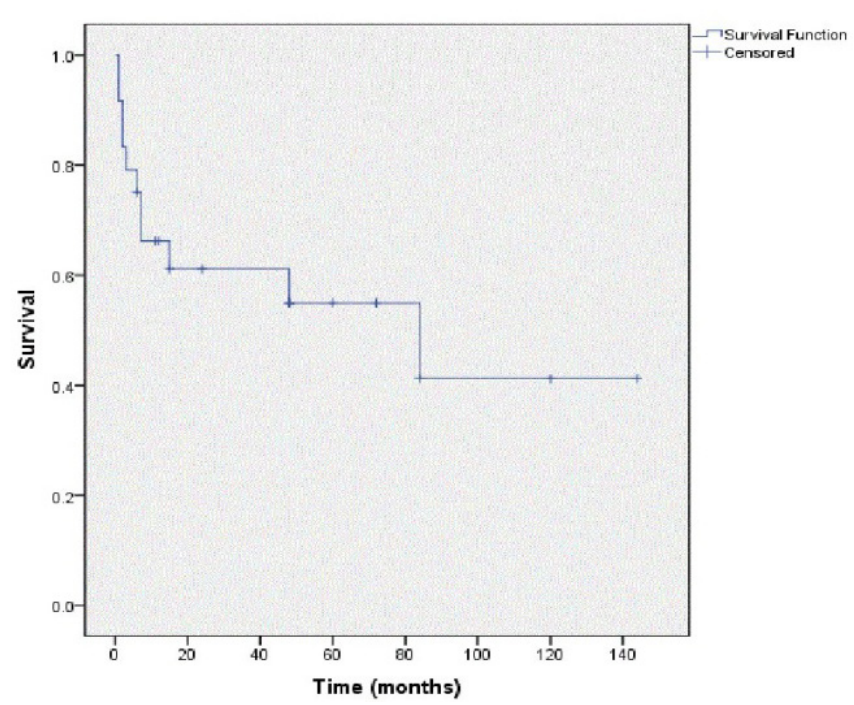

Fig. 1. Probability of survival

Using the prognostic index for AIDS-associated KS mortality proposed by Stebbing [8] , we found that $30 \%(8 / 27)$ of our patients were high risk as judged by his score (score $>12$ points) and only $7 \%(2 / 27)$ were patients with low risk (score $<5$ points). In individuals with lowrisk prognostic scores, medium-risk and high risk, respectively, the probability of survival at 1 -year was $1.0,0.76$ and 0.5 , respectively.

\section{Discussions}

In our study KS was diagnosed in young patients with a median age of 42 years, with advanced HIV disease (AHD), with a median CD4 cell count of $101 \mathrm{cells} / \mathrm{mm}^{3}$. Between January 2012 and December 2013, in Bucharest, Romania, $37 \%$ of the patients notified with HIV infection had AHD [9].

KS has been associated with HHV-8 infection, with the virus being always present in the tumor [10]. HHV-8 infection and KS are more common among HIV-positive men who have sex with men (MSM) or bisexual men, women who were infected with HIV through sex with bisexual men.

In a case-control study performed in Portugal, the mean age at diagnosis of AIDS-defining cancers (ADCs) was 41 years, and the mean CD 4 cell count was 278 cells $/ \mathrm{mm}^{3}$; KS was the most frequent (61\%) of ADCs [11].

A recent study has reported a high incidence of Kaposi sarcoma during the first 3 months of ART, describing KS as one of the manifestations of immune reconstitution inflammatory syndrome (IRIS) [[12]. In our study, the median time from ART initiation to KS diagnosis was 5 months (IQR 0-39); roughly half of our patients who were on ART (10/19) were in virological failure at the time of KS diagnosis and in the other 9/19 patients KS could have been an IRIS manifestation.

Patients in this study presented with extended mucocutaneous lesions and disseminated visceral involvement and $59 \%$ had poor-risk KS, a similar proportion to that reported in limited resource countries [13,14].

The overall mortality in the study group was $41 \%$, associated with a short time span from KS diagnosis to death, of six months, similar to the pre-ART era. This is in part due to the fact that over half of the patients were staged as poor-risk (ACTG). All patients who died were classified as having poor-risk KS. Out of the other five patients classified as poor-risk KS, four had a favorable outcome and one was lost to follow-up. All patients who died had one or more opportunistic infections; tuberculosis, Cytomegalovirus and Pneumocystis carinii infection were the most frequent.

Our patients had poor access to specific therapy for KS. Only two patients received interferon and one received liposomal doxorubicin. Three patients received local radiotherapy. Poor access to specific therapy places us at the same level as Sub-Saharan Africa.

Overall cumulative survival was $69 \%$ at 1 year and $64 \%$ at 2 years, similar data being reported from a primary care HIV programme in South Africa [15]; however, the survival in our study proved to be lower compared to data reported from Chelsea and Westminster Hospital in London, UK [16].

Using Stebbing's prognostic index for AIDS-associated KS [8] regarding the probability of survival, we found similar values compared to Stebbing's study.

HAART can also lead to complete resolution of KS lesions in patients with good immunological response and limited disease [17]. Simultaneous systemic chemotherapy is indicated in patients with advanced, symptomatic KS [18]. The positive effect of ART on survival in patients with AIDS-associated KS is well known [19]. A large study of 326 individuals with AIDS-KS also found that mortality was not influenced by the initiation of ART before development of KS [6], similar to the data derived from our study group. In Stebbing's study [8], 80\% of the patients had KS as the first AIDS-defining illness. KS as the first AIDS-defining illness and a higher CD4 counts were significantly associated with a better survival. In our study, in which $30 \%$ of the patients had KS as the first defining illness, mortality was significantly associated with CD4 count under 200 cells $/ \mathrm{mm}^{3}$.

The retrospective nature and the small number of patients are intrinsic limitations; since gastrointestinal and/ or pulmonary involvements are usually asymptomatic in HIV-related KS [20,21], the rate of organ involvement may have been clinically underdiagnosed. However, our results describe an aspect of the clinical picture for latepresenter HIV-positive patients diagnosed with KS, with severe evolution and rapid progression of disease.

\section{Conclusion}

In our HIV population, KS remains an important cause of morbidity and mortality especially in late presenters. The high mortality rate in this study population was as- 
sociated with poor immunological status, extended KS and high incidence of opportunistic infections. Poor access to specific systemic treatment may have contributed to the high mortality rate and remains an important challenge in our clinic. For patients with disseminated, progressive or symptomatic disease, chemotherapy along HAART is an important component of the treatment.

\section{Conflict of interest}

None to declare

\section{References}

1. Gao SJ, Kingsley L, Hoover DR, et al. Seroconversion to antibodies against Kaposi's sarcoma-associated herpesvirus-related latent nuclear antigens before the development of Kaposi's sarcoma. N Engl J Med. 1996; 335(4):233.

2. Engels E, Biggar RJ, Hall H, et al. Cancer risk in people infected with human immunodeficiency virus in the United States. Int J Cancer. 2008; 123:187-194.

3. Rose LJ, Harris JE, Fishman AD, et al. Kaposi Sarcoma clinical presentation. http://emedicine.medscape.com/article/279734-clinical. [accessed 28.01.2015]

4 Simard EP, Pfeiffer RM, Engels EA. Cumulative incidence of cancer among individuals with acquired immunodeficiency syndrome in the United States. Int J Cancer. 2011;117:1089-1096.

5. European Center for Diseases Control. HIV/AIDS surveillance in Europe 2008-2013. http://ecdc.europa.eu/en/ [accessed 28.01.2015]

6. Krown SE, Metroka C, Wernz JC. Kaposi's sarcoma in the acquired immune deficiency syndrome: a proposal for uniform evaluation, response, and staging criteria. AIDS Clinical Trials Group Oncology Committee. J Clin Oncol. 1989;7(9):1201-7.

7. Mitsuyasu RT. Clinical variants and staging of Kaposi's sarcoma. Semin Oncol. Jun. 1987;14(2Suppl3):13-8
8. Stebbing J, Sanitt A, Nelson M, et al. A prognostic index for AIDS associated Kaposi's sarcoma in the era of highly active antiretroviral therapy. Lancet. 2006;367:1495-502.

9. Jipa RE, Manea E., Benea S, et al. Characteristics of late presenters in Bucharest. J Int AIDS Soc. 2014;17(Suppl 3):19691.

10. Laney AS, Cannon MJ, Jaffe HW, et al. Human herpes virus 8 presence and viral load are associated with the progression of AIDS-associated Kaposi's sarcoma. AIDS. 2007;21:1541-1545.

11. Sofia Fernandes L. Human immunodeficiency virus and cancer. A population of HIV-infected patients at Hospital de Santa Maria and predictors of cancer. GERMS. 2012;2:60-74.

12. Lacombe JM, Boue F, Grabar S, et al. Risk of Kaposi sarcoma during the first months on combination antiretroviral therapy. AIDS. 2013;27:635-43.

13. Agaba PA, Sule HM, Ojoh Ro, et al. Presentation and survival of patients with AIDS-related Kaposi's sarcoma in Jos, Nigeria. Int J STD AIDS. 2009;20:410-413

14. Phipps W, Sewankambo F, Nguyen, et al. Gender differences in clinical presentation and outcomes of epidemic Kaposi Sarcoma in Uganda. PLoS ONE. 2010.5:e13936. doi:10.1371/journal.pone.0013936.

15. K. Chu., G. Mahlangeni, S. Swannet, et al. AIDS-associated Kaposi's sarcoma is linked to advanced disease and high mortality in a primary care HIV programme in South Africa. J Int AIDS Soc. 2010;13:23.

16. Bower M, Dalla Pria A, Coyle C, et al. Prospective stage-stratified approach to AIDS-related Kaposi's sarcoma. J Clin Oncol. 2014;32:40914.

17. Martelotta F, Berretta M, Vacher E, et al. AIDS- Related Kaposi Sarcoma: State of the ART and therapeutic Strategies. Current HIV Research. 2009; 7:634-638.

18. Krown SE, Northfelt DW, Osoba D, et al. Use of liposomal anthracyclines in Kaposi's sarcoma. Semin Oncol. 2004;31:36-52.

19. Di Lorenzo G, Konstantinopoulos PA, Pantanowitz L, et al. Management of AIDS-related Kaposi's sarcoma. Lancet Oncol. 2007;8:167-176.

20. Kibria R, Siraj U, Barde C. Kaposi sarcoma of the stomach and duodenum in human immunodeficiency virus infection. Dig Endosc. 2010;22:241-2.

21. Borie R, Cadranel J, Guihot A, Marcelin AG, et al. Pulmonary manifestations of human herpesvirus-8 during HIV infection. Eur Respir J. 2013;42:1105-18 